\title{
Vaginal hysterectomy vs. laparoscopically assisted vaginal hysterectomy in women with symptomatic uterine leiomyomas: a retrospective study
}

\author{
Ewelina Litwińska ${ }^{1}$, Marek Nowak², Dorota Kolasa-Zwierzchowska², Anna Nowińska-Serwach², \\ Jacek Władziński², Artur Szpakowski², Marian Szpakowski², Jacek R. Wilczyński², Tomasz Stetkiewicz ${ }^{2}$ \\ ${ }^{1}$ Perinatology and Gynecology Department, Polish Mother's Memorial Hospital-Research Institute, tódź, Poland \\ ${ }^{2}$ Gynecology and Gynecologic Oncology Department, Polish Mother's Memorial Hospital-Research Institute, Łódź, Poland
}

\begin{abstract}
Introduction: Uterine leiomyomas are the most common benign tumors of the female reproductive system. Although the majority of myomas are asymptomatic, some patients have symptoms or signs of varying degrees and require a hysterectomy.

The aim of the study was to compare the clinical results of two minimally invasive hysterectomy techniques: vaginal hysterectomy $(\mathrm{VH})$ and laparoscopically assisted vaginal hysterectomy (LAVH).

Material and methods: A retrospective, observational study was performed at a tertiary care center: the Gynecology and Gynecologic Oncology Department, Polish Mother's Memorial Hospital Research Institute. The study period was from January 2003 to December 2012. A total of 159 women underwent either vaginal hysterectomy $(\mathrm{VH}, n=120)$ or laparoscopically assisted vaginal hysterectomy $(\mathrm{LAVH}, n=39)$ for symptomatic uterine myomas. Outcome measures, including past medical history, blood loss, major complications, operating time and discharge time were assessed and compared between the studied groups. Statistical analysis was performed using Student $t$-test, $U$-Mann Whitney test, $\chi^{2}$ test and Yates ${ }^{\prime} \chi^{2}$ test. $P<0.05$ was considered statistically significant.

Results: There were no differences in patients' mean age. Parity was significantly higher in the VH group (VH $1.9 \pm 0.7$ vs. LAVH $1.5 \pm 0.8 ; p=0.008$ ). No difference was found in the mean \pm standard deviation (SD) uterine volume between vaginal hysterectomy and LAVH groups (179 \pm 89 vs. $\left.199 \pm 88 \mathrm{~cm}^{3}\right)$, respectively. The mean operative time was significantly longer for the LAVH group ( $83 \pm 29$ vs. $131 \pm 30 \mathrm{~min} ; p=0.0001)$. The intraoperative blood loss (VH $1.3 \pm 1.1$ vs. LAVH $1.4 \pm 0.9 \mathrm{~g} / \mathrm{dl} ; p=0.2$ ) and the rate of intra- and postoperative complications were similar in both groups studied. The mean discharge time was longer for LAVH than for VH (VH $4.2 \pm 1.2$ vs. LAVH $5.3 \pm 1.3$ days, $p=0.0001)$

Conclusions: Laparoscopically assisted vaginal hysterectomy and $\mathrm{VH}$ are safe hysterectomy techniques for women with the myomatous uterus. Concerning the LAVH, the abdominal-pelvic exploration and the ability to perform adnexectomy safely represent the major advantages comparing with $\mathrm{VH}$. Vaginal hysterectomy had a shorter operating time and the mild blood loss making it a suitable method of hysterectomy for cases in which the shortest duration of surgery and anesthesia is preferable.
\end{abstract}

Key words: uterine myomas, vaginal hysterectomy (VH), laparoscopically assisted vaginal hysterectomy $(\mathrm{LAVH})$.

\section{Introduction}

Uterine myomas are the most common benign tumors in women. In most cases, myomas are asymptomatic but they can also cause a variety of symptoms including: menometrorrhagia, pelvic pain, pressure complaints (constipation, pollakiuria) [1]. Large fibroids can be a reason for subfertility and can complicate the course of pregnancy and delivery.

The standard method in treatment of uterine myomas is still operative treatment. The basic gynecological procedure used in cases of symptomatic myomas is hysterectomy. It is one of the most common gynecological operations in the world. In the United States, about 600000 hysterectomies are performed annually, most of them because of benign tumors of the reproductive system [2-5]. Efforts are made to reduce intra- and postoperative morbidity concentrating on both reduction in the overall number of hysterectomies as well as the development of additional techniques alternative to abdominal hysterectomy. Nowadays, there are a few different methods of hysterectomy: abdominal hysterectomy $(\mathrm{AH})$, supracervical hysterectomy and less in- 
vasive techniques such as vaginal hysterectomy (VH), laparoscopically assisted vaginal hysterectomy (LAVH), supracervical (SLH) and total laparoscopic hysterectomy (TLH). There is no consensus among the gynecologists regarding the optimal route of hysterectomy in patients with an enlarged, myomatous uterus. The choice of the appropriate method is based on clinical and technical criteria (the size of the uterus, earlier vaginal deliveries, abdominal and vaginal operations) [6]. In a few studies comparing the techniques of hysterectomy in patients with an enlarged uterus, both operation and recovery time proved to be shorter in the vaginal route of hysterectomy than in the abdominal route [7-9]. Advantages of hysterectomy in laparoscopic assistance include the reduction in postoperative pain and shorter hospital stay in comparison to abdominal hysterectomy [10,11].

So far, only a few studies have concentrated on the comparison of less invasive techniques of hysterectomy. The aim of the study was to compare LAVH with $\mathrm{VH}$ in a retrospective analysis as well as to evaluate the intra- and postoperative complications and the time of postoperative hospitalization.

\section{Material and methods}

A retrospective observational study was conducted. The study population consisted of patients operated at a tertiary care center: the Gynecology and Gynecologic Oncology Department, Polish Mother's Memorial Hospital Research Institute due to symptomatic myomas. The study period was from January 2003 to December 2012. Patients who underwent vaginal hysterectomy or laparoscopically assisted vaginal hysterectomy were selected from the computer system, where files of all operations performed in the Department are stored. A group of 159 patients was selected and their documentation was reviewed. Outcome measures, including past medical history (previous labors and operations), blood loss, major intra- and postoperative complications, size of the uterus, histopathological diagnosis, operating time and total hospital stay were assessed and compared between the groups. Patients were divided into two groups depending on the type of the operation performed: VH group, $n=120$, LAVH group, $n=39$.

Described operative procedures were always conducted in the same way and by the same operating team. Before the planned operation the clinical analysis of the uterus and its mobility was performed as well as ultrasound examination of the uterus with special emphasis on the size, number and localization of myomas and the size of the uterus. In the presence of indications, the diagnostic hysteroscopy or endometrial sampling was performed.

The type of anesthesia was adapted to the hysterectomy route: $\mathrm{VH}$ was performed under regional and LAVH under general anesthesia.

\section{Operative technique}

Vaginal hysterectomy began with infiltration of the vaginal wall with saline and circular incision of the vagina around the cervix as described by Hefni et al. [12]. After that, the bladder was pushed upwards. In sequence rectovaginal and vesicouterine space were opened. Bilaterally uterosacral ligaments and uterine vessels were clamped, cut and ligated. If the uterine size did not allow easy exteriorization, bisecting, coring, enucleation of myomas or combinations of these volume-reducing techniques were performed. The next step was clamping, cutting and ligation of proper ovarian ligaments, round ligaments and fallopian tubes. Closure of the vaginal vault was done with continuous suture. At the end, sacrospinous colpopexy was performed [12]. During the LAVH procedure, three trocars were introduced: one trocar for optics and other two for laparoscopic instruments. Bipolar coagulation and scissors were used. To move the uterus the manipulator was inserted into uterus through the vagina. Laparoscopically assisted vaginal hysterectomy included coagulation and sectioning of the round ligament, proper ovarian ligaments and fallopian tubes. For adnexectomy, bilaterally infundibulopelvic ligaments were coagulated and cut. The next step was opening of the bladder flap and bladder dissection. The laparoscopic procedure included also coagulating and transecting of the uterine vessels. Vaginal phases included circular incision of the vagina around the cervix and preparation upwards as well as opening of the rectovaginal and vesicouterine space [12]. The next step was bilateral clamping, cutting and ligation of cardinal and uterosacral ligament. Coring, enucleation of myomas was done if necessary. Closure of the vaginal vault concluded the vaginal phase, at which time the pelvis and the abdomen were reevaluated through the laparoscope to be sure of hemostasis.

The analysis of intra- and postoperative complications included: the duration of the operation (from the first incision to placement of the last suture), the volume of the uterus, frequency of conversions to an abdominal hysterectomy and the mean blood loss. Blood loss was estimated by comparing the hemoglobin level 24 hours before the operation and 3 days after the operation. The uterine volume was calculated in a ViewPoint system.

\section{Statistical analysis}

In the statistical analysis, Student's $t$-test for independent samples (for quantitative variances which distribution fulfilled the assumptions of normality), Mann-Whitney $U$ test (for quantitative variances which distribution did not fulfill the assumptions of normality), $\chi^{2}$ test (for qualitative parameters compared in two populations) and $\chi^{2}$ test with Yates correction (for qual- 
itative parameters compared in two populations which did not fulfill the minimal number condition). The normality of distribution was checked with KolmogorovSmirnov test. $P$ value $<0.05$ was statistically significant.

\section{Results}

The comparison of characteristics between patients who underwent VH and LAVH is shown in Table I. There was no difference in patients' mean age. Patients in the $\mathrm{VH}$ group delivered more times than patients in the LAVH group (VH $1.9 \pm 0.7$ vs. LAVH $1.5 \pm 0.8 ; p=0.008)$. Patients in the LAVH group underwent more often cesarean section or other abdominal operations than patients in the $\mathrm{VH}$ group (cesarean section $6.6 \% \mathrm{vs}$. $23 \%$, operations $30 \%$ vs. $48 \%, p<0.05)$. The indication for surgery in both groups was symptomatic myomas. No difference was found in the mean uterus volume between vaginal hysterectomy and LAVH groups $\left(\mathrm{VH} 179.3 \pm 88\right.$ vs. LAVH $\left.199.9 \pm 88 \mathrm{~cm}^{3} ; p=0.2\right)$.

The size of the uterus had an influence on the duration of the operation $(p=0.005)$. This correlation was particularly expressed in the VH group ( $p=0.001$ ) (Fig. 1). A strong correlation was also observed between the size of the uterus and the intraoperative blood loss $(p=0.048)$. This correlation was stronger in the $\mathrm{VH}$ group $(p=0.028)$ (Fig. 2). Pearson index was used to determine the correlations.

The mean operating time was significantly shorter in the VH group than in the LAVH group $(83 \pm 29$ vs. $131 \pm 30 \mathrm{~min} ; p=0.0001)$. No difference was found in the mean intraoperative blood loss between vaginal hysterectomy and LAVH groups (VH $1.3 \pm 1.1$ vs. LAVH $1.4 \pm 0.9 \mathrm{~g} / \mathrm{dl} ; p=0.2$ ). Adnexectomy was performed in $66.6 \%$ of patients in the LAVH group and in $33.3 \%$ of patients in the $\mathrm{VH}$ group $(p<0.05)$. Total hospital stay was significantly longer in the LAVH group (VH $4.2 \pm 1.2$ vs. LAVH $5.3 \pm 1.3$ days; $p=0.0001$ ) (Table II).

Table III shows the comparison of intra- and postoperative complications in both groups. There was no statistically significant difference between groups in frequency of major complications (bladder laceration, excessive hemorrhage, abdominal wall hematoma). In both compared groups, one patient required a preoperative blood transfusion due to anemia. In both groups, two patients needed postoperative blood transfusions due to an intraoperative hemorrhage. Two cystotomies occurred in the $\mathrm{VH}$ group and one in the LAVH group. Bladder laceration was recognized intraoperatively and sutured during operation. Conversion to laparotomy

Tab. I. Baseline characteristics

\begin{tabular}{lccc}
\hline Factor & VH $(n=120)$ & LAVH $(n=39)$ & $p$ \\
\hline Age & $50.9 \pm 7.7$ & $50.2 \pm 6.9$ & 0.635 \\
\hline Parity & $1.9 \pm 0.7$ & $1.5 \pm 0.8$ & 9.008 \\
\hline Previous cesarean section & $8(6.6 \%)$ & 0.004 \\
\hline Previous pelvic and abdominal surgery & $36(30 \%)$ & $19(48 \%)$ & 0.0328 \\
\hline
\end{tabular}

Data are presented as mean \pm standard deviation or as $n$ (\%).

$\mathrm{VH}$ - vaginal hysterectomy, LAVH - laparoscopically assisted vaginal hysterectomy

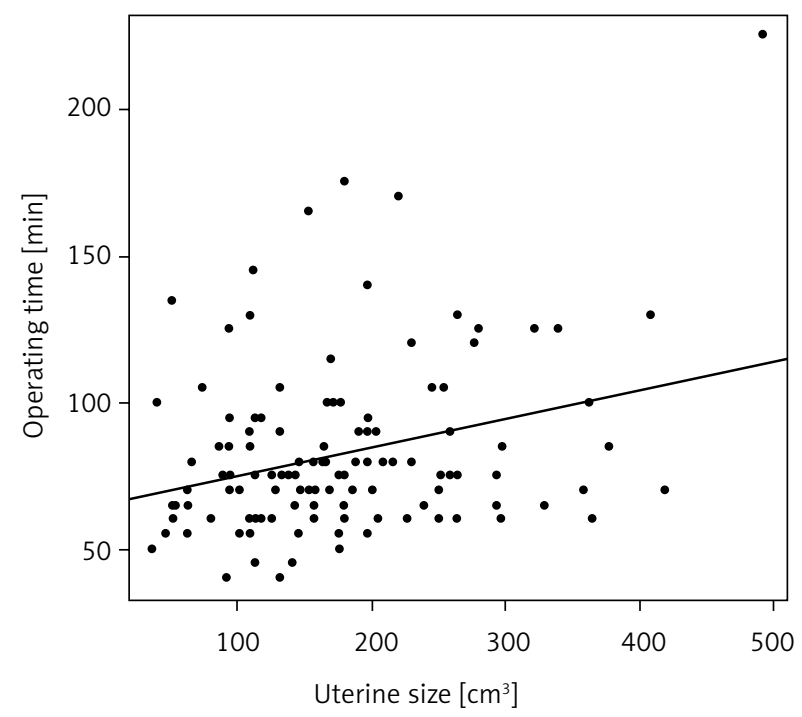

Fig. 1. The correlation between the size of the uterus and the duration of the operation in the $\mathrm{VH}$ group

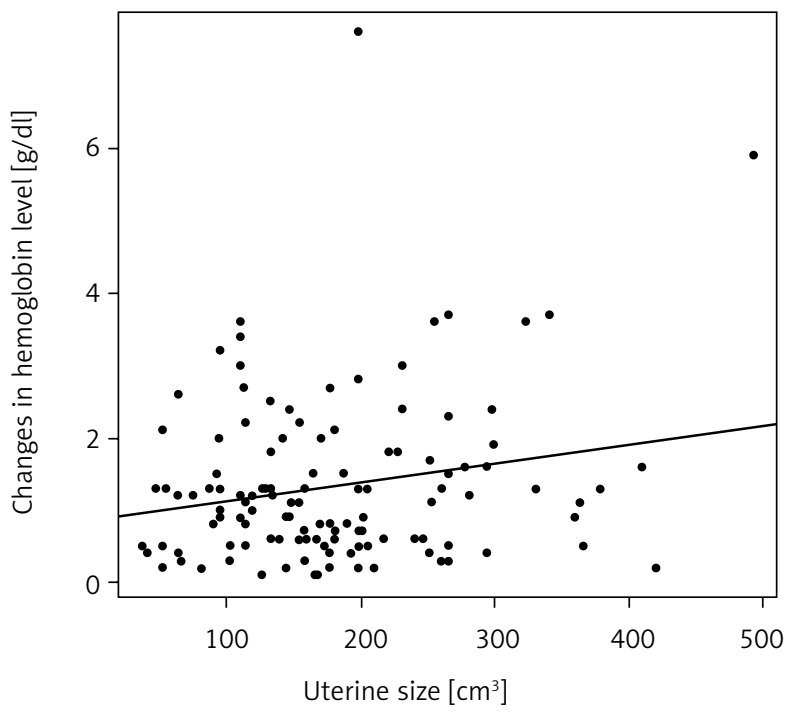

Fig. 2. The correlation between the size of the uterus and the intraoperative blood loss in the $\mathrm{VH}$ group 
Tab. II. Intra- and postoperative characteristics

\begin{tabular}{lccc}
\hline Factor & VH $(n=120)$ & LAVH $(n=39)$ & $p$ \\
\hline Operating time $(\mathrm{min})$ & $83 \pm 29$ & $131 \pm 30$ & 0.0001 \\
\hline Uterus volume $\left(\mathrm{cm}^{3}\right)$ & $179.3 \pm 88$ & $199.9 \pm 88$ & 0.208 \\
\hline Adnexectomy & $40(33.3 \%)$ & $26(66.6 \%)$ & 0.0002 \\
\hline Estimated blood loss $(\mathrm{g} / \mathrm{dl})$ & $13.5 \pm 11.5$ & $14.7 \pm 9.2$ & 0.222 \\
\hline Postoperative hospital stay (days) & $4.2 \pm 1.2$ & $5.3 \pm 1.3$ & 0.0001 \\
\hline
\end{tabular}

Data are presented as mean \pm standard deviation or as $n(\%)$.

$\mathrm{VH}$ - vaginal hysterectomy, LAVH - laparoscopically assisted vaginal hysterectomy

Tab. III. Complications

\begin{tabular}{lccc}
\hline Complication & VH $(n=120)$ & LAVH $(n=39)$ & $p$ \\
\hline Conversion to laparotomy & 2 & 1 & 0.7493 \\
\hline Bladder laceration & 2 & 1 & 0.7493 \\
\hline Postoperative blood transfusion & 2 & 2 & 0.5414 \\
\hline Abdominal wall hematoma & 0 & 1 & 0.5526 \\
\hline
\end{tabular}

VH - vaginal hysterectomy, LAVH - laparoscopically assisted vaginal hysterectomy

was required in two patients in the $\mathrm{VH}$ group and one patient in the LAVH group (in the $\mathrm{VH}$ group the reason was bleeding from infundibulopelvic ligament, in the LAVH group the reason was the bladder laceration).

\section{Discussion}

The choice of the most efficient technique of hysterectomy is still very controversial. Prospective studies point out that patients who underwent LAVH have a shorter postoperative hospital stay, are less painful, and have a shorter recovery time in comparison to patients who underwent an abdominal hysterectomy [11, 13].

There is also no advantage in performing LAVH when $\mathrm{VH}$ is possible [14].

In a prospective analysis comparing LAVH and $\mathrm{VH}$, Summit et al. presented data which showed a clear connection between LAVH and longer operating time as well as more significant blood loss and need for analgesics [15]. In a similar study, Soriano et al. also emphasized a longer operating time in patients who underwent LAVH. The need for analgesics and the postoperative recovery time was similar in both groups [16]. Harkki et al., after reviewing 13885 hysterectomies, stated that patients planned to undergo LAVH are at an increased risk $(2.2 \%)$ of urinary tract lesions in comparison to patients planned to undergo $\mathrm{VH}(0.4 \%)$ [17]. Authors of the eVALuate study which prospectively evaluated $\mathrm{LAVH}$ and $\mathrm{VH}$ stated a similar risk of intraoperative complications in both procedures [11].

In our Department, myomas of the uterus accounted for $62 \%$ of indications for LAVH. $53 \%$ of all VHs were performed because of symptomatic myomas.
In the analyzed groups, the operating time was shorter in the VH group. Previous studies also described a shorter operating time in $\mathrm{VH}$ without laparoscopic assistance $[16,18]$. The amount of blood loss in the described study differs from previously described. The mean blood loss defined as a difference in hemoglobin levels on a day before operation and three days after operation was compared in patients who underwent LAVH and VH. According to our authors, the blood loss was less significant in the $\mathrm{VH}$ group than in the LAVH group [18]. No significant differences were stated in the amount of main intra- and postoperative complications. It is consistent with the results of the eVALuate study [11]. Previous statements about the postoperative recovery time after $\mathrm{VH}$ and $\mathrm{LAVH}$ are unclear. In the presented study, patients who underwent LAVH had a longer recovery time in comparison to patients who underwent $\mathrm{VH}$. In the literature, some authors prove that LAVH is connected with a longer recovery time, other studies show that the time of the postoperative hospital stay is comparable in both groups $[11,14,18]$. Women who were suspected to be more problematic were qualified for LAVH and that is the reason why they stayed under longer supervision. The patients were discharged home on a normal diet, fully mobile, without elevated temperature or analgesics.

Contrary to some studies available in the literature, which clearly emphasize the advantage of the $\mathrm{VH}$ over LAVH we stated that due to duration of LAVH as well as a low percentage of intra- and postoperative complications, this method can be found as fully acceptable [16]. Laparoscopically assisted vaginal hysterectomy appears to be a more efficient technique in the case of patients planned to have a simultaneous adnexectomy 
because of benign ovarian tumors. Laparoscopically exssisted vaginal hysterectomy was more frequently performed in patients with expected intraoperative difficulties caused by a history of pelvic inflammatory disease, operations within the pelvis and abdomen as well as endometriosis.

Meta-analysis of randomized trials conducted by Johnson et al. defined $\mathrm{VH}$ as a preferable method of hysterectomy in the case of favorable safety conditions $[19,20]$. However, VH has its limitations such as technical difficulties during adnexectomy. In our opinion, as to the short operation time and low blood loss, $\mathrm{VH}$ remains a method of choice in elderly patients with risk factors. This technique is the most frequent one in our Department.

The review of the largest studies analyzing the techniques of hysterectomy in the case of myomas shows that the vaginal operations remain the most frequent procedures. The main advantages of the LAVH in comparison to $\mathrm{VH}$ are the possibility to explore the abdomen and pelvis as well as performing a safe adnexectomy, as far there are no specified indications for each of the hysterectomy technique. However, the purpose of LAVH is to widen the possibilities of conducting less invasive procedures for more indications such as ovarian tumors, ovarian and oviductal adhesions, endometriosis, previous operations within the abdomen and pelvis, rather than displacing $\mathrm{VH}$.

\section{Conclusions}

1. The main advantages of the LAVH in comparison to the $\mathrm{VH}$ are the possibility to explore the abdomen and pelvis as well as performing a safe adnexectomy.

2. Vaginal hysterectomy is characterized by the shortest operating time as well as low blood loss. That is the reason why it is the most appropriate when the short time of anesthesia and operation is required.

\section{Disclosure}

Authors report no conflicts of interest.

\section{References}

1. Su WH, Lee WL, Cheng MH, et al. Typical and atypical clinical presentation of uterine myomas. J Chin Med Assoc 2012; 75: 487-493.
2. Wilcox LS, Koonin LM, Pokras R, et al. Hysterectomy in the United States 1988-1990. Obstet Gynecol 1994; 83: 549-555.

3. Lepine LA, Hillis SD, Marchbanks PA, et al. Hysterectomy surveillance United States, 1980-1993. MMWR CDC Surveill Summ 1997; 46: 1-15.

4. Keshavarz H, Hillis SD, Kiele BA, et al. Hysterectomy surveillance - United States, 1994-1999. MMWR CDC Surveill Summ 2002; 51 (SS-5): 1-8.

5. Wu JM, Wechter ME, Geller EJ, et al. Hysterectomy rates in the United States, 2003. Obstet Gynecol 2007; 110: 1091-1095.

6. Garry R. The future of hysterectomy. BJOG 2005; 112: 133-139.

7. Benassi L, Rossi T, Kaihura CT, et al. Abdominal or vaginal hysterectomy for enlarged for enlarged uteri: a randomized clinical trial. Am J Obstet Gynecol 2002; 187: 1561-1565.

8. Taylor SM, Romero AA, Kammerer-Doak DN, et al. Abdominal hysterectomy for enlarged myomatosus uterus compared with vaginal hysterectomy with morcellation. Am J Obstet Gynecol 2003; 189: 1579-1583.

9. Harmanli $\mathrm{OH}$, Gentzler $\mathrm{CK}$, Byun $\mathrm{S}$, et al. A comparison of abdominal and vaginal hysterectomy for the large uterus. Int J Gynaecol Obstet 2004; 87: 19-23.

10. Hwang JL, Seow KM, Tsai YL, et al. Comparative study of vaginal, laparoscopically assisted vaginal and abdominal hysterectomies for uterine myoma larger than $6 \mathrm{~cm}$ in diameter or uterus weighting at least 450 g: a prospective randomized study. Acta Obstet Gynecol Scand 2002; 81: 1132-1138.

11. Garry R, Fountain J, Mason S, et al. The eVALuate study: two parallel randomised trials one comparing laparoscopic with abdominal hysterectomy, the other comparing laparoscopic with abdominal hysterectomy, the other comparing laparoscopic with vaginal hysterectomy. BMJ 2004; 328: 129-136.

12. Hefni MA, El-Toukhy TA. Sacrospinous colpopexy at vaginal hysterectomy: method, results and follow up in 75 patients. J Obstet Gynecol 2000; 20: 58-62.

13. Falcone T, Paraiso MFR, Wascha E. Prospective randomized clinical trial of laparoscopically assisted vaginal hysterectomy versus total abdominal hysterectomy. Am J Obstst Gynecol 1999; 180: 955-962.

14. Darai E, Soriano D, Kimata P, et al. Vaginal hysterectomy for enlarged uteri, with or without laparoscopic assistance: randomized study. Obstet Gynecol 2001; 97: 712-716.

15. Summit RL, Stovall TG, Lipscombe GH, et al. Randomized comparison of laparoscopic-assisted vaginal hysterectomy with standard vaginal hysterectomy in an outpatient setting. Obstet Gynecol 1992; 80: 895-899.

16. Soriano D, Goldstein A, Lecuru F, et al. Recovery from vaginal hysterectomy compared with laparoscopy-assisted vaginal hysterectomy: a prospective, randomized, multicenter study. Acta Obstet Gynecol Scand 2001; 80: 337-341.

17. Harkki P, Kurki T, Sjoberg J, et al. Safety aspects of laparoscopic hysterectomy. Acta Obstet Gynecol Scand 2001; 80: 383-391.

18. Sesti F, Ruggeri V, Pietropolli A, et al. Laparoscopically assisted vaginal hysterectomy versus vaginal hysterectomy for enlarged uterus. JSLS 2008; 12: 246-251.

19. Johnson N, Barlow D, Lethaby A, et al. Methods of hysterectomy: systematic review and meta-analysis of randomized controlled trials. BMJ 2005; 330: 1478-1481.

20. Johnson N, Barlow D, Lethaby A, et al. Surgical approach to hysterectomy for benign gynaecological disease. Cochrane Database Syst Rev 2006; 2: CD003677. 